\title{
Ring chromosome 22 syndrome
}

INSERM

\section{Source}

INSERM. (1999). Orphanet: an online rare disease and orphan drug data base. Ring chromosome 22 syndrome. ORPHA:1446

Ring chromosome 22 syndrome is an autosomal anomaly characterized by variable clinical features, most commonly including global developmental delay, hypotonia, growth retardation with microcephaly, intellectual disability with severe speech delay, seizures or abnormal EEG, autistic spectrum disorder and other behavioral characteristics. 